\title{
Una aplicación de métodos de conocimiento base y clasificación difusa para predecir calidad de agua en tres comunas del sur de Chile
}

\author{
An application of knowledge based method and fuzzy sets to predict basin water quality \\ in three southern counties of Chile
}

\author{
Gerardo Vergara $^{a *}$ y Jorge Gayoso ${ }^{b}$ \\ *Autor de correspondencia: anstituto de Investigación Forestal, Fundo Teja Norte s/n, Valdivia, Chile, \\ tel. 56-63-211476, gvergara@infor.cl \\ bUniversidad Austral de Chile, Facultad de Ciencias Forestales, Instituto de Manejo Forestal, Valdivia, Chile.
}

\begin{abstract}
SUMMARY
In spite of several international efforts promoting quality of surface water through low sediment generation, this issue has not been undertaken in Chile. Environmental management can be a complex matter due to a deficiency in information or knowledge about causal relationships. This study shows applicability of spatial analysis techniques and Knowledge-Based Systems for modeling probability of supply of free-sediment waters in different areas of three southern counties in Chile. The method considered selection of variables through experts' opinion and the application of Analytic Hierarchy Process (AHP), where physical and social variables were standardized for the analysis to be carried out. A free-sediment water probability map was produced through fuzzy sets (membership functions) of every spatial unit. The model validation was carried out by turbidity measures in streams. These measures indicated a correlation between low turbidity areas (high quality waters) and forested, low access and good drainage zones. These maps constitute a supporting tool for the sustainable development of fish farming, fly fishing and tourism activities.
\end{abstract}

Key words: spatial analysis, water quality, fuzzy sets.

\section{RESUMEN}

A pesar de las numerosas iniciativas internacionales que promueven prácticas para minimizar la producción de sedimentos y cuidar la calidad de las aguas superficiales en el territorio, en Chile este es aún un tema pendiente. El desconocimiento de relaciones causales o carencias de información sistémica y agregada dificulta en muchos casos la adecuada toma de decisiones. Este estudio demuestra la aplicabilidad de técnicas de análisis espacial y modelación basada en el conocimiento disponible de factores del territorio, para estimar la probabilidad de generación de agua libre de sedimentos sobre $2.547,6 \mathrm{~km}^{2}$ en cuencas de tres comunas del sur de Chile. El método comprendió la selección de variables mediante consulta a expertos y la utilización del proceso analítico jerárquico. Diferentes atributos fisicosociales fueron categorizados para realizar el análisis. Esta información se integró para cada unidad de área estudiada mediante un modelo que empleó clasificación difusa (funciones de pertenencia), generando un mapa de probabilidad de producción de agua libre de sedimentos. Se comprobó la validez del modelo mediante mediciones directas de turbidez en los cauces. Estos mapas pueden ser de utilidad para planificar el desarrollo sustentable de actividades como acuicultura, pesca deportiva y turismo.

Palabras clave: análisis espacial, calidad de agua, clasificación difusa.

\section{INTRODUCCIÓN}

El desarrollo de importantes actividades en el sur de Chile, tales como el suministro de agua potable, la acuicultura, la pesca deportiva y el turismo en general, demanda de forma creciente agua de alta calidad en ríos, lagos y estuarios y, en consecuencia, el resguardo de los ecosistemas que la proveen. $\mathrm{Al}$ mismo tiempo se han intensificado las actividades asociadas a los usos agropecuario, forestal y de generación hidroeléctrica, incrementando las presiones sobre la red hidrológica, lo que ha dado surgimiento a los primeros conflictos entre usuarios.
Actualmente existe un mayor conocimiento de cómo funcionan los ecosistemas en términos de la interrelación de sus componentes y los impactos potenciales sobre éstos. Se ha comprobado que la calidad del agua puede ser afectada por diversas actividades que se desarrollan en las áreas tributarias de la cuenca, tales como la agricultura, el madereo, el uso del fuego, el pastoreo y la construcción de caminos, entre otras. Sin embargo, muchos de estos impactos potenciales pueden ser amortiguados o incrementados dependiendo de las características físicas que presenta el territorio circundante. Es así como diferentes estudios han demostrado que el grado de cobertura vegetal 
-determinado por el uso del suelo- es uno de los factores más relevantes que condiciona el escurrimiento superficial $\mathrm{y}$, consecuentemente, los efectos indeseados sobre los cursos de agua (Keithley 2000, NCWAP 2001). También se ha demostrado la importancia de mantener el bosque junto a los cauces, lo cual puede contribuir fuertemente a la calidad de las aguas debido a su efecto "filtro" que actúa sobre sedimentos y contaminantes desde sitios tributarios (Durst y Ferguson 2000).

Por otro lado, existe consenso respecto a que factores tales como deficiencias en la localización, construcción y mantenimiento de caminos pueden constituir una de las mayores fuentes no puntuales en aportes de sedimentos hacia los cursos de agua (Lee et al. 2004, USDA Forest Service 1999, Beaudry et al. 2003). Este potencial para degradar la calidad del agua viene desde caminos ubicados sobre laderas escarpadas y cruce de caminos sobre cursos de agua. En Estados Unidos, investigaciones han demostrado que el $90 \%$ de los sedimentos que terminan en las aguas de tierras forestales están asociados al diseño y mantenimiento de caminos (Daniels et al. 2004). Estos hechos indican que la modelación de procesos de erosión y, por lo tanto, el cálculo del aporte de sedimentos hacia los cauces es una tarea que puede llegar a ser altamente compleja por tratarse de un proceso multivariante y multitemporal influenciado por las relaciones espaciales de los elementos del territorio.

Boyd y Wainger (2003) destacan la necesidad del análisis espacial en la evaluación de los servicios ecosistémicos, debido a que tanto las funciones biofísicas de producción como los factores sociales de los mismos dependen del patrón de paisaje en el cual surgen. Los índices espaciales constituyen además la base necesaria para la realización de cualquier valoración económica posterior (CCAD-PNUD/ GEF 2002, Barton et al. 2003, Boyd y Wainger 2003, Banzhaf y Boyd 2004, Boyd 2004).

Entre las opciones de análisis que utilizan información espacial y manejan incertidumbre, la literatura destaca los sistemas basados en el conocimiento, el proceso analítico jerárquico y las técnicas de agrupamiento difuso.

Los sistemas basados en el conocimiento o también llamados conocimiento base se han utilizado para la evaluación ecológica $-y$ por ende de los sistemas hídricos- como apoyo a la gestión del territorio (Reynolds et al. 1996, Schmoldt y Rauscher 1996). Dicha forma de enfrentar el manejo de recursos y la toma de decisiones representa un esquema útil para identificar, por ejemplo, áreas prioritarias de producción, ya que encapsula la evaluación multivariante y sus relaciones, de modo que ayuda a su análisis y la modificación o actualización del proceso. Esto es particularmente válido para modelación hidrológica, ya que permite readecuar o ajustar los modelos en la medida que el conocimiento de los ecosistemas mejora en el tiempo.
El proceso analítico jerárquico (AHP) es una técnica de manejo de la incertidumbre a través del método de comparación de pares, desarrollada por Saaty (1977), de amplia aplicación y particularmente útil en el contexto de la selección y pertinencia de variables que se utilizan en los modelos hidrológicos.

La clasificación (lógica) de grupos difusos es una técnica para integrar y clasificar el conocimiento múltiple con ciertos niveles de incertidumbre (Zadeh 1965). Debido a que los grupos difusos pueden manejar información aproximada de una forma sistemática, se ha aplicado para modelar tópicos abstractos o complejos tales como aptitud de hábitats o la condición de cuencas que dependen de numerosas y diversas condiciones y donde la información imprecisa es común (Dai et al. 2004, Ocampo-Duque et al. 2006). Esta técnica constituye un medio de clasificación integrador de las variables utilizadas en los modelos. Por ejemplo, permite el análisis y clasificación de las variables que afectan procesos hidrológicos, las cuales generalmente son factores de carácter continuo tales como la distancia a los caminos, las pendientes y la cobertura vegetal.

Este trabajo tiene por objetivo demostrar la aplicabilidad de una técnica de análisis espacial y modelación para apoyar la gestión del territorio mediante la aplicabilidad de técnicas para resolver problemas de tipo ambiental donde las variables son de distinta naturaleza (continuas, discretas, ordinales, categóricas) y tienen asociados diversos niveles de incerteza. Para ello, comprende la utilización de clasificación difusa y del proceso analítico jerárquico bajo el marco de los sistemas basados en el conocimiento, para generar índices sintéticos de la estimación de la probabilidad de conservar agua libre de sedimentos en cauces, durante época de lluvias, para tres comunas del sur de Chile.

La hipótesis de trabajo considera que un modelo construido en base a información de variables de estado del territorio siguiendo las metodologías propuestas, es capaz de simular con suficiente sensibilidad la probabilidad de provisión de agua de calidad y discriminar entre áreas con diferente potencial.

\section{MÉTODOS}

Área de estudio. El área de estudio comprende tres comunas (Pucón, Lanco y Corral) de las Regiones de La Araucanía y Los Ríos en Chile. Estas comunas presentan marcadas diferencias en aspectos de geomorfología, distribución de los usos de la tierra y grado de influencia antrópica (cuadro 1).

Esquema metodológico. La metodología utilizada para la evaluación y proposición de indicadores consideró el conocimiento base (knowledge base method) para el sistema de apoyo a la decisiones (Reynolds et al. 1996, Schmoldt y Rauscher 1996) mediante la aplicación de las técnicas de 
Cuadro 1. Atributos de las comunas del área de estudio.

Characteristics for counties of the study area.

\begin{tabular}{llll}
\hline \multirow{2}{*}{ Atributo } & \multicolumn{1}{c}{ Comuna } \\
\cline { 2 - 4 } & \multicolumn{1}{c}{ Pucón } & \multicolumn{1}{c}{ Lanco } & \multicolumn{1}{c}{ Corral } \\
Localización & Cordillera andina & Depresión Intermedia & Cordillera de la Costa \\
Superficie $\left(\mathrm{km}^{2}\right)$ & $1.248,5$ & 532,4 & 766,7 \\
Altitud máxima $(\mathrm{m})$ & 600 & 400 & 2.000 \\
Relieve & Relieve abrupto, cordones & Planicies y cerros de altitud & Relieve abrupto marcado por \\
& montañosos y volcanes & media & la Cordillera de la Costa \\
Suelos (drenaje) & Rápido o moderado rápido & Moderado y moderado a lento & Moderado a lento \\
Precipitaciones (mm/año) & 1.000-2.500 & $1.600-1.800$ & 2.000-2.400 \\
Uso del suelo & Bosque nativo: 60 & Bosque nativo: 31 & Bosque nativo: 68 \\
$(\%$ de la comuna) & Agroganadero: 18 & Agroganadero: 50 & Agroganadero: 2,5 \\
& Plantaciones: 1 & Plantaciones: 16 & Plantaciones: 20 \\
Población (habitantes) & Total: 21.170 & Total: 15.107 & Total: 5.463 \\
& Rural: 7.270 & Rural: 4.724 & Rural: 1.793 \\
\hline
\end{tabular}

análisis jerárquico (AHP) y clasificación difusa y, por otro lado, la aplicación de la Combinación Lineal Ponderada (WLC por sus siglas en inglés) para la integración de los indicadores parciales. De esta forma, la obtención de índices de probabilidad de producción de agua limpia comprendió las etapas descritas en la figura 1.

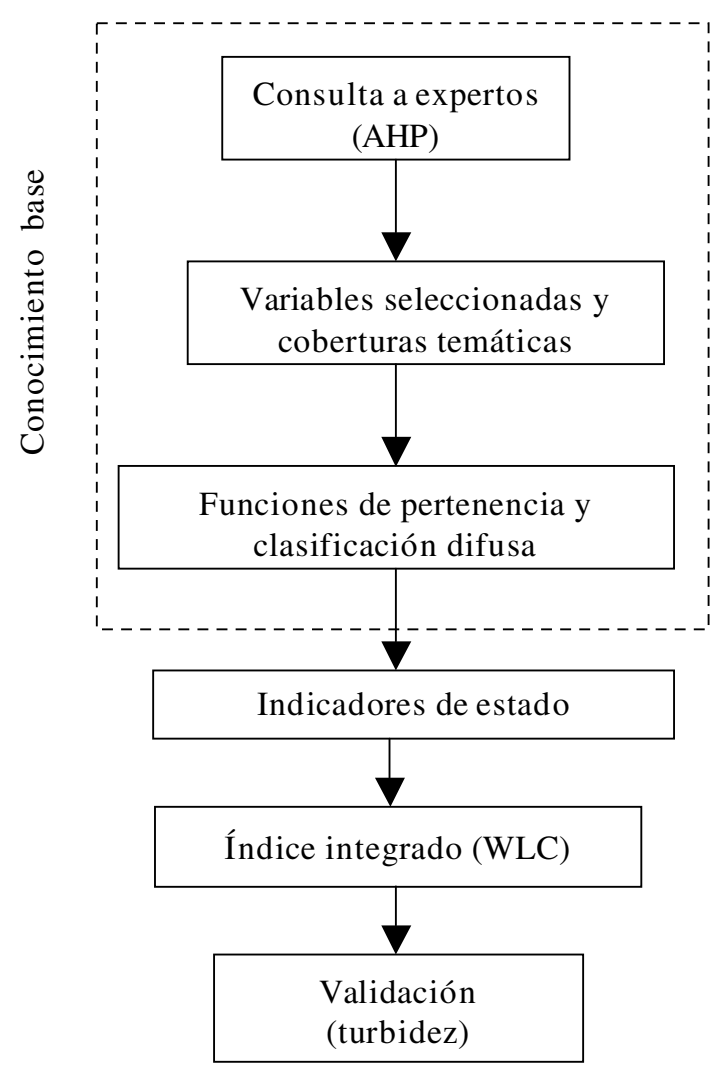

Figura 1. Esquema metodológico general utilizado en el estudio.

Methodological approach used in the study.
Los análisis generales de las bases de datos -asociadas a coberturas de sistemas de información- se realizaron con planillas de cálculo Excel 2002. Para el manejo y análisis de la información digital georreferenciada se utilizó Arcview GIS 3.2, ArcGIS 8.0 e Idrisi Kilimanjaro.

Selección de variables con consulta a expertos. Para realizar esta etapa participaron 46 expertos en el área de suelos, hidrología y manejo de cuencas a quienes se consultó acerca de las variables de mayor importancia en la generación de agua de calidad en el territorio mediante los pasos considerados en la metodología del AHP. Las variables originales propuestas fueron tamaño y localización del recurso hídrico, temporalidad o permanencia, uso del suelo de áreas tributarias, superficie de las unidades vegetales, tipo de explotaciones, composición y cobertura vegetacional, distancia de los caminos al cauce, cruce de cauces, tamaño de propiedad, pendiente, drenaje del suelo y densidad de caminos. El propósito de esta fase fue identificar y conocer la ponderación de las variables a utilizar en el estudio.

Esta etapa se realizó mediante una matriz de comparación de pares de cada variable, con los cuales se elaboró una razón de consistencia de las respuestas de cada experto (Saaty 1977). La razón de consistencia (CR) indica la probabilidad de que la matriz de evaluación sea generada aleatoriamente, por tanto, aquellas con CR mayores a 0,05 fueron descartadas del análisis.

Elaboración de coberturas temáticas. Una vez definidas las variables, se procedió a recolectar esta información para llevarla a formatos cartográficos. Es por ello que el estudio se basó principalmente en información digital georreferenciada. Dicha información en formato vectorial ${ }^{1}$

\footnotetext{
1 Esta información proviene originalmente de distintos formatos, es decir, líneas, polígonos y puntos, y debió ser traspasada a un formato raster común para su análisis.
} 
consideró las coberturas del catastro de bosques nativos para Chile (CONAF y CONAMA 1999b), la digitalización del trabajo de ordenamiento del suelo para la zona sur del país (Schlatter et al. 1995, 1997), así como de las coberturas de límites comunales (INE), roles de propiedad (SII), red hidrológica (DGA) y redes camineras (MOPT).

También se derivaron nuevas coberturas de las relaciones espaciales de los atributos de estos temas. Es el caso de los buffers alrededor de la red hidrológica conteniendo el tipo de uso de suelo, la cobertura de cercanía de caminos a los cursos de agua, la cobertura de cruce de caminos sobre cursos de agua y la cobertura que indica la densidad de caminos por unidad de área.

En esta etapa la información disponible en formato vectorial se convirtió a formato raster tomando como base las imágenes satelitales Landsat TM. De esta forma la unidad mínima de la información final es un píxel de 30 por 30 metros de superficie y se logra además que la información sea comparable y posible de procesar mediante la aplicación de clasificación difusa de estas variables. Estas variables se clasificaron en dos tipos, de acuerdo a su ubicación espacial e importancia en relación a los cauces (cuadro 2).

Las variables identificadas mediante la consulta a expertos permitieron definir cuáles incluir en el análisis. Los pesos derivados del AHP en base a las estimaciones de los consultados se han agregado también al cuadro 2 . Las variables que los expertos excluyeron fueron la temporalidad de los cursos de agua y la forma y tamaño de las unidades vegetales.

Clasificación difusa de los indicadores. En esta etapa se convirtió la información disponible a imágenes con una escala probabilística en un rango de 0 a 1 mediante el módulo Fuzzy de Idrisi. Los valores cercanos a 1 indican una alta posibilidad (probabilidad) de que se produzca agua de calidad. La confiabilidad de los mapas obtenidos depende de los parámetros aplicados durante la transformación, la cual es controlada por una función de pertenencia. Para este estudio se utilizaron los supuestos descritos en el cuadro 3.

Muestreo de campo y validación. Para la verificación de cada una de las zonas de generación de agua se diseñó un muestreo para determinar la turbidez del agua ${ }^{2}$ que contenía la red hidrológica en un punto determinado. Para ello se establecieron puntos de muestreo en las áreas de confluencia de dos o más cursos de agua en las tres comunas en estudio. El área de aporte hasta este punto se definió mediante la extensión ArcWater de ArcGIS.

2 Según la OMS (Organización Mundial para la Salud), la turbidez del agua para consumo humano no debe superar en ningún caso las 5 FTU, y estará idealmente por debajo de 1 FTU.
Las mediciones de turbidez fueron realizadas después de un periodo de lluvias (postevento) con el fin de estimar los impactos sobre el suelo que alteran la calidad del agua en los cauces. Estas mediciones fueron realizadas mediante un turbidímetro portátil marca Hanna HI 93703 que basa sus mediciones haciendo pasar un haz de luz infrarroja a través de un vial conteniendo la muestra a medir. Mediante esta forma se verificó la correspondencia entre el mapa generado y los valores medidos en terreno, relacionando la calidad de las aguas con las variables que caracterizan la cuenca de provisión.

Una de las razones de utilizar la turbidez como elemento de control es su facilidad de medición de campo, proveyendo además una forma de evaluación visual del estado de la calidad del recurso. Sin embargo, este tipo de evaluación no pretende ser un indicador de la calidad global del agua, ya que existen compuestos que en altas concentraciones no alteran los niveles de turbidez, en particular en zonas de producción agrícola-ganadera.

\section{RESULTADOS}

Los resultados del proceso de clasificación difusa incluyen los indicadores de estado. Estos son representados por las variables definidas por el AHP, las cuales fueron traducidas a imágenes de posibilidad para cada una de las comunas en estudio. Dichos indicadores de posibilidad representan una variable de interés bajo una perspectiva espacial, lo cual ayuda a identificar las zonas del territorio donde estas variables pueden afectar la calidad del agua en los cauces (figura 2).

Un factor determinante en los procesos de erosión y transporte de sedimentos corresponde a la pendiente del terreno (Sheridan y So 2001). El valor o posibilidad de este indicador señala cuáles áreas son más propensas a generar sedimentos. En este caso, las tonalidades oscuras muestran áreas de bajas pendientes $\mathrm{y}$, por tanto, de menor riesgo a producir efectos negativos sobre la calidad del agua.

De la misma forma, se produjeron los indicadores de estado para todas las demás variables consideradas y para cada una de las comunas. El resumen de estos indicadores se presenta en el cuadro 4.

Los indicadores parciales identificados constituyen en sí una forma individual de evaluar el estado general de los ecosistemas y, por tanto, determinan a la vez su grado de deterioro y el riesgo a perder su potencial de generación de agua de calidad. Además, la clasificación de estas variables mediante técnicas difusas constituye una forma de aproximación para determinar la verdadera naturaleza de éstas y sus efectos sobre la producción de agua de calidad. Sin embargo, es ampliamente necesaria una mayor comprensión científica de los alcances y efectos de los cambios en amplitud de estas variables, con el objetivo de mejorar estos modelos. 
Cuadro 2. Tipo y descripción de los indicadores.

Description and type of indicators.

\begin{tabular}{|c|c|c|}
\hline Tipo de indicador & $\begin{array}{c}\text { Nombre } \\
\text { (Ponderación) }\end{array}$ & Descripción - unidad \\
\hline \multirow[t]{3}{*}{$\begin{array}{l}\text { Zona de manejo de } \\
\text { cauces (ZMC) }\end{array}$} & $\begin{array}{l}\text { Calidad ZMC } \\
\qquad(20 \%)\end{array}$ & $\begin{array}{l}\text { Creados mediante isobandas de } 25 \mathrm{~m} \text { a cada lado de los cursos de agua, para luego } \\
\text { intersectar estas áreas con la cobertura de uso del suelo de cada comuna. }\end{array}$ \\
\hline & $\begin{array}{l}\text { Cruce de cauces } \\
\qquad(4 \%)\end{array}$ & $\begin{array}{l}\text { Éste incluye la intersección de la cobertura de caminos (MOPT) de cada comuna con } \\
\text { la cobertura hidrológica de esta misma zona. }\end{array}$ \\
\hline & $\begin{array}{l}\text { Distancia a caminos } \\
\qquad(7 \%)\end{array}$ & $\begin{array}{l}\text { Corresponde a la distancia lineal que existe entre los caminos y los cursos de agua, } \\
\text { para lo cual se generaron áreas de isodistancia. }\end{array}$ \\
\hline \multirow[t]{5}{*}{ Áreas tributarias } & $\begin{array}{l}\text { Cobertura vegetal } \\
\qquad(24 \%)\end{array}$ & $\begin{array}{l}\text { Se consideró la clasificación del catastro vegetacional chileno (CONAF y CONAMA } \\
\text { 1999a) para cada comuna. }\end{array}$ \\
\hline & $\begin{array}{l}\text { Tamaño propiedad } \\
\qquad(4 \%)\end{array}$ & Se clasificaron los predios de cada comuna de acuerdo a su superficie. \\
\hline & $\begin{array}{l}\text { Pendiente terreno } \\
\qquad(18 \%)\end{array}$ & De acuerdo a la inclinación del terreno definida por un modelo digital de elevación. \\
\hline & $\begin{array}{l}\text { Drenaje suelo } \\
\qquad(16 \%)\end{array}$ & $\begin{array}{l}\text { Identifica áreas de similar respuesta en el drenaje interno de los suelos de cada comuna. } \\
\text { Las categorías fueron definidas considerando el trabajo de Schlatter et al. (1997). }\end{array}$ \\
\hline & $\begin{array}{c}\text { Densidad caminos } \\
\qquad(7 \%)\end{array}$ & Se clasificó la cantidad lineal de caminos por unidad de superficie. \\
\hline
\end{tabular}

Cuadro 3. Funciones de pertenencia para los indicadores seleccionados.

Membership functions for selected indicators.

\begin{tabular}{|c|c|c|c|}
\hline Indicador & $\begin{array}{l}\text { Función de } \\
\text { pertenencia }\end{array}$ & Tendencia & Niveles/categorías \\
\hline Calidad ZMC & $\begin{array}{l}\text { Lineal } \\
\text { monotónica } \\
\text { creciente }\end{array}$ & $\begin{array}{l}\text { Aumentos en el grado de cobertura del suelo alrededor de los } \\
\text { cursos de agua reducen en forma significativa los posibles impactos } \\
\text { de las actividades productivas (Gallo et al. 2005). }\end{array}$ & $\begin{array}{l}\text { De acuerdo a la cobertura vegetal: } \\
\text { sin cobertura permanente, muy abierto, } \\
\text { abierto, semidenso y denso. }\end{array}$ \\
\hline $\begin{array}{l}\text { ruce de } \\
\text { uces }\end{array}$ & $\begin{array}{l}\text { Sigmoidal } \\
\text { monotónica } \\
\text { decreciente }\end{array}$ & $\begin{array}{l}\text { Expresa los valores de número de cruce de cauces por kilómetro } \\
\text { cuadrado y están basados en los estudios realizados por Beaudry } \\
\text { et al. (2003). En este caso, valores bajo } 0,25 \text { cruces } / \mathrm{km}^{2} \text { producen } \\
\text { efectos marginales sobre la calidad del agua, mientras que valores } \\
\text { superiores a } 1,0 \text { cruces } / \mathrm{km}^{2} \text { tendrán una fuerte influencia sobre } \\
\text { la calidad del recurso. }\end{array}$ & $\begin{array}{l}0-0,25 ; 0,25-0,6 ; 0,6-0,8 ;>0,8 \text { cruces } / \\
\mathrm{km}^{2} .\end{array}$ \\
\hline $\begin{array}{l}\text { Distancia } \\
\text { caminos }\end{array}$ & $\begin{array}{l}\text { Sigmoidal } \\
\text { monotónica } \\
\text { creciente }\end{array}$ & $\begin{array}{l}\text { Refleja la distancia de la fuente generadora de sedimento; mientras } \\
\text { más alejado se encuentran los caminos menor será la probabilidad } \\
\text { de que estos alcancen los cauces (Elliot et al. 1998). }\end{array}$ & $\begin{array}{l}0-100,100-250,250-500,500-1.000 \mathrm{y} \\
>1.000 \mathrm{~m} .\end{array}$ \\
\hline $\begin{array}{l}\text { Cobertura } \\
\text { vegetal }\end{array}$ & $\begin{array}{l}\text { Lineal } \\
\text { monotónica } \\
\text { creciente }\end{array}$ & $\begin{array}{l}\text { Aumentos en el grado de cobertura del suelo alrededor de los } \\
\text { cursos de agua reducen en forma significativa los posibles impactos } \\
\text { de las actividades productivas (Gallo et al. 2005). }\end{array}$ & $\begin{array}{l}\text { Sin cobertura permanente, muy abierto, } \\
\text { abierto, semidenso y denso. }\end{array}$ \\
\hline $\begin{array}{l}\text { Tamaño } \\
\text { propiedad }\end{array}$ & $\begin{array}{l}\text { Forma de J } \\
\text { monotónica } \\
\text { creciente }\end{array}$ & $\begin{array}{l}\text { Se estimó que los propietarios que poseen menor superficie } \\
\text { utilizan prácticas menos amigables con el medio ambiente } \\
\text { debido a que poseen menor acceso a las tecnologías y en muchas } \\
\text { situaciones solo realizan una agricultura de subsistencia (Morales } \\
\text { y Parada 2005). }\end{array}$ & $\begin{array}{l}0-100 ; 100-250 ; 250-500 ; 500-1.000 \mathrm{y} \\
>1.000 \text { ha. }\end{array}$ \\
\hline $\begin{array}{l}\text { Pendiente } \\
\text { terreno }\end{array}$ & $\begin{array}{l}\text { Sigmoidal } \\
\text { monotónica } \\
\text { decreciente }\end{array}$ & $\begin{array}{l}\text { Aumentos en los niveles de pendiente del terreno hacen más } \\
\text { vulnerables a los cursos de agua, por efectos de erosión o } \\
\text { transporte de sedimentos (Sheridan y So 2001). }\end{array}$ & $0-10 ; 10-15 ; 10-30 ; 30-45$ у > 45\%. \\
\hline Drenaje suelo & $\begin{array}{l}\text { Lineal } \\
\text { monotónica } \\
\text { creciente }\end{array}$ & $\begin{array}{l}\text { Aumentos de densidad suelo, le sigue un aumento en escorrentía } \\
\text { y por tanto de transporte de sedimentos hacia los cauces (Hilliard } \\
\text { y Reedyk 2000). }\end{array}$ & $\begin{array}{l}\text { Lento, moderado a lento, moderado, } \\
\text { moderado a rápido y rápido. }\end{array}$ \\
\hline $\begin{array}{l}\text { Densidad } \\
\text { caminos }\end{array}$ & $\begin{array}{l}\text { Lineal } \\
\text { monotónica } \\
\text { decreciente }\end{array}$ & $\begin{array}{l}\text { A medida que aumenta la cantidad de caminos por unidad } \\
\text { de superficie se espera una reducción cada vez mayor en los } \\
\text { niveles de calidad de agua (Gallo et al. 2005). }\end{array}$ & $2 ; 2-4 ; 4-8 ; 8-12$ y > 12 metros/ha. \\
\hline
\end{tabular}




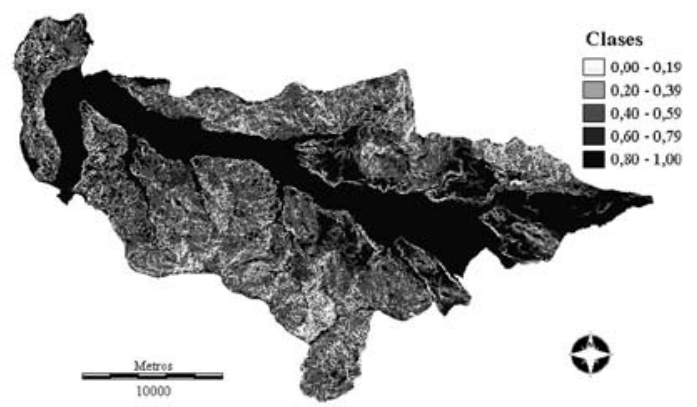

Figura 2. Indicador de niveles de posibilidad de pendiente del terreno en la comuna de Lanco.

Ground slope possibility index of the Lanco County.

Cuadro 4. Distribución de la superficie comunal según índice de posibilidad y variable control.

Surface distribution by county related to possibility index and control variable.

\begin{tabular}{|c|c|c|c|c|}
\hline \multirow{2}{*}{ Variable } & \multirow{2}{*}{ Categorías } & \multicolumn{3}{|c|}{ Comunas } \\
\hline & & Corral & Lanco & Pucón \\
\hline \multirow{5}{*}{$\begin{array}{l}\text { Pendiente del } \\
\text { terreno }\end{array}$} & $0-0,2$ & 26,7 & 42,2 & 0,5 \\
\hline & $0,2-0,4$ & 11,5 & 5,3 & 11,8 \\
\hline & $0,4-0,6$ & 35,2 & 32,4 & 33,7 \\
\hline & $0,6-0,8$ & 19,5 & 14,5 & 13,5 \\
\hline & $0,8-1,0$ & 7,0 & 5,5 & 40,4 \\
\hline \multirow{5}{*}{$\begin{array}{l}\text { Calidad } \\
\text { ZMC }\end{array}$} & $0-0,2$ & 0,42 & 8,4 & 0,3 \\
\hline & $0,2-0,4$ & 10,6 & 23,1 & 53,1 \\
\hline & $0,4-0,6$ & 31,5 & 22,7 & 31,1 \\
\hline & $0,6-0,8$ & 7,7 & 8,9 & 3,2 \\
\hline & $0,8-1,0$ & 49,6 & 36,6 & 12,0 \\
\hline \multirow{5}{*}{$\begin{array}{l}\text { Distancia a } \\
\text { caminos }\end{array}$} & $0-0,2$ & 1,8 & 24,5 & 2,0 \\
\hline & $0,2-0,4$ & 8,3 & 34,2 & 7,0 \\
\hline & $0,4-0,6$ & 19,5 & 19,2 & 12,5 \\
\hline & $0,6-0,8$ & 20,5 & 9,4 & 17,3 \\
\hline & $0,8-1,0$ & 49,9 & 12,6 & 61,2 \\
\hline \multirow{5}{*}{$\begin{array}{l}\text { Cobertura } \\
\text { vegetal }\end{array}$} & $0-0,2$ & 7,0 & 10,0 & 17,1 \\
\hline & $0,2-0,4$ & 17,0 & 56,7 & 17,5 \\
\hline & $0,4-0,6$ & 8,9 & 3,1 & 4,6 \\
\hline & $0,6-0,8$ & 52,4 & 18,4 & 15,5 \\
\hline & $0,8-1,0$ & 14,6 & 11,6 & 45,3 \\
\hline \multirow{5}{*}{$\begin{array}{l}\text { Tamaño de } \\
\text { la propiedad }\end{array}$} & $0-0,2$ & 15,9 & 35,2 & 5,3 \\
\hline & $0,2-0,4$ & 14,4 & 21,4 & 0,7 \\
\hline & $0,4-0,6$ & 5,0 & 13,9 & 0 \\
\hline & $0,6-0,8$ & 3,8 & 18,7 & 1,9 \\
\hline & $0,8-1,0$ & 60,9 & 10,7 & 92,1 \\
\hline \multirow{5}{*}{$\begin{array}{l}\text { Drenaje } \\
\text { del suelo }\end{array}$} & $0-0,2$ & 15,9 & 10,5 & 0 \\
\hline & $0,2-0,4$ & 75,5 & 57,7 & 0 \\
\hline & $0,4-0,6$ & 8,5 & 31,7 & 0 \\
\hline & $0,6-0,8$ & 0 & 0 & 76,8 \\
\hline & $0,8-1,0$ & 0 & 0 & 23,2 \\
\hline \multirow{5}{*}{$\begin{array}{l}\text { Densidad de } \\
\text { caminos }\end{array}$} & $0-0,2$ & 0 & 45,9 & 0 \\
\hline & $0,2-0,4$ & 20,7 & 15,8 & 0 \\
\hline & $0,4-0,6$ & 33,7 & 19,9 & 26,2 \\
\hline & $0,6-0,8$ & 26,9 & 12,3 & 22,0 \\
\hline & $0,8-1,0$ & 18,5 & 5,9 & 51,8 \\
\hline
\end{tabular}

Indicadores integrados. La integración mediante WLC en Arcview GIS 3.2, dio como resultado el valor integrado producto de la combinación de los diferentes factores y sus pesos derivados del AHP. Las imágenes representan con tonalidades más claras aquellas áreas de mayor probabilidad potencial de generar agua de calidad y con tonalidades oscuras aquellas zonas que presentan características que indican mayor riesgo para la producción de este servicio ecosistémico.

Estas zonas identifican la agregación de los diferentes indicadores parciales. Los valores altos de probabilidad coinciden con los valores más altos de posibilidad de las variables generadas mediante clasificación difusa. Estas zonas potenciales para generar agua de calidad están en áreas de mayor presencia de bosques, con menor acceso y suelos de mejor drenaje. Sin embargo, un aspecto a destacar es el potencial integrador de las variables por parte del modelo, en particular desde la dimensión espacial de los indicadores.

Para la comuna de Corral se presentan niveles altos de probabilidad en la sección sur y sureste del territorio, coincidiendo con la presencia de la Reserva Forestal Valdivia y zonas con menor influencia de caminos y vegetaciones más densas (figura 3). En el área de Lanco se encuentran ubicadas en los extremos norte, sur y este de la comuna, mientras que en Pucón los niveles altos de probabilidad se presentan en gran parte del territorio, lo cual es coincidente con la existencia de las reservas y parques nacionales de esta zona y las actividades de menor impacto que se desarrollan en la comuna. Junto a ello, para Pucón se debe destacar la existencia de asociaciones vegetales más densas que en las otras comunas del estudio.

Por otro lado, la comuna de Pucón presenta la mayor parte de su territorio con altas probabilidades para la provisión potencial de agua de calidad (niveles mayores a 0,6); sólo en las áreas de menor pendiente en las cercanías de la ciudad se presentan menores probabilidades. Estos resultados son concordantes con la demanda actual de recursos hídricos en la comuna de Pucón, la que requiere calidad de aguas superficiales para sostener las crecientes actividades de turismo y recreación, tales como la pesca deportiva, deportes acuáticos y usos del agua con y sin contacto directo. Lanco presenta una mayor fragmentación de áreas con altos niveles de probabilidad, donde algunos núcleos de los niveles más altos se concentran en las áreas de mayor altitud y más apartadas de la comuna. Generalmente, estas áreas están asociadas a zonas sin acceso debido a las características morfológicas del sector, lo que ha impedido la realización de actividades productivas (figura 4).

Bajo esta perspectiva, Pucón es la comuna que presenta un mayor potencial de producción de agua de calidad, representado por las imágenes antes expuestas. Corral es la segunda comuna en potencial, mientras que, en la comuna de Lanco, se identifican serios problemas de sedimentación en los cursos de agua después de un evento de precipitaciones. 

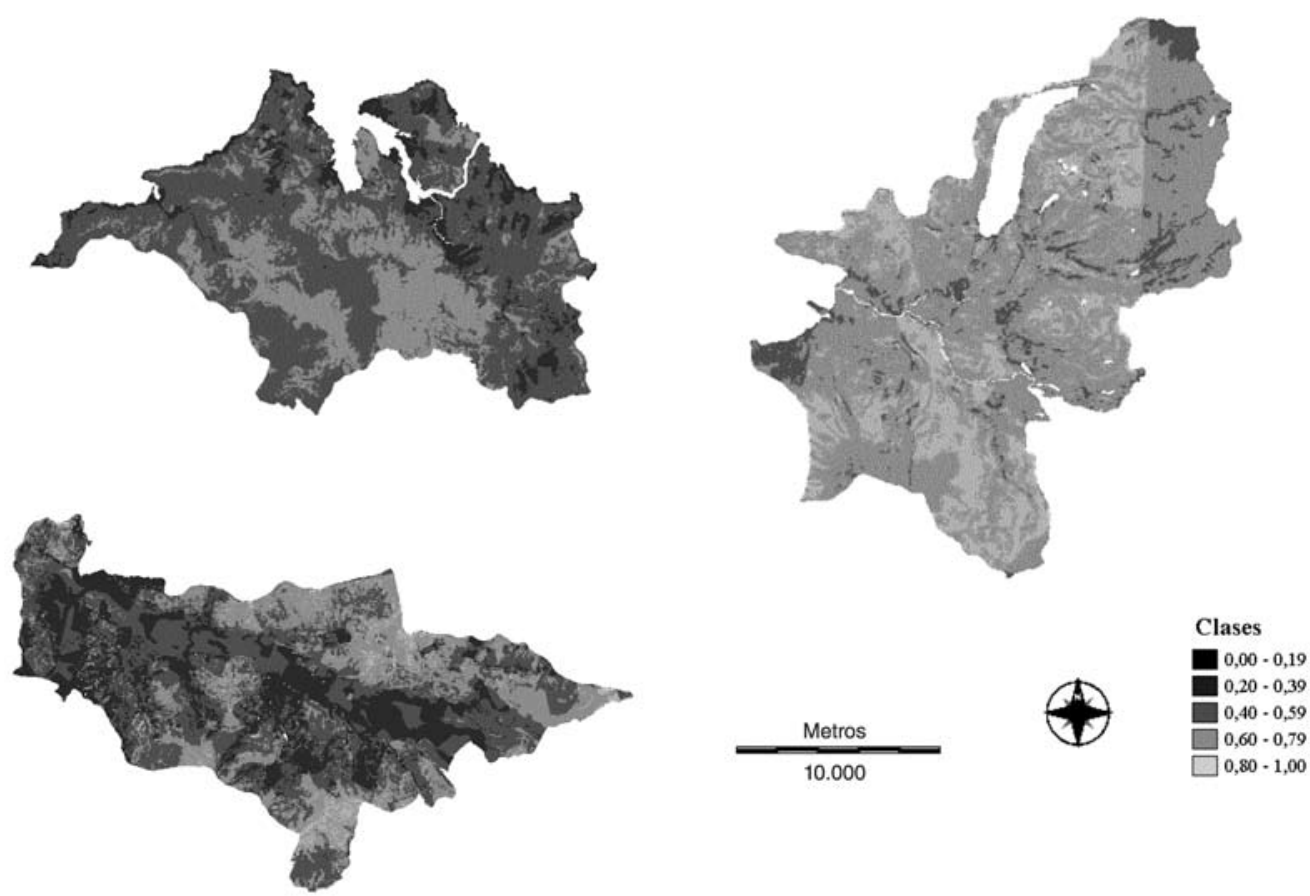

Figura 3. Índice de posibilidad para las comunas de Corral (superior izquierda), Pucón (superior derecha) y Lanco (inferior). Possibility index images for Corral (upper left), Pucón (upper right) and Lanco Counties (lower).

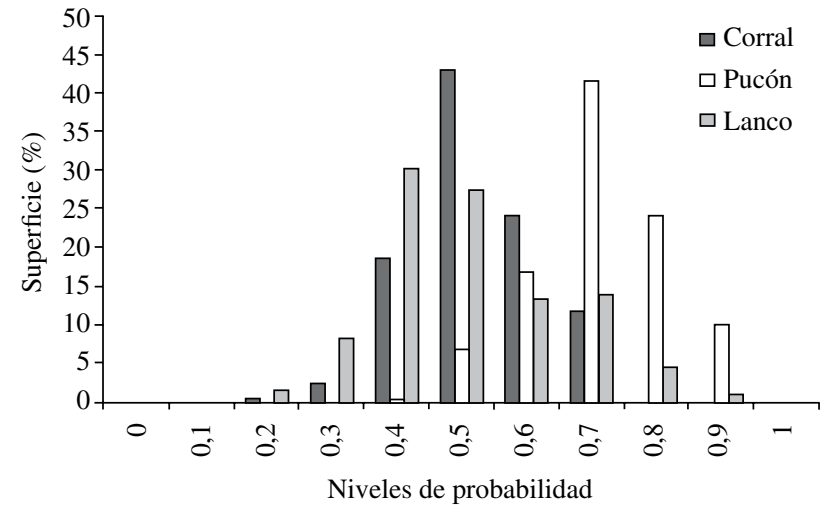

Figura 4. Niveles de posibilidad de las comunas de Corral, Pucón y Lanco.

Possibility levels for Corral, Pucón and Lanco Counties.

Como una forma de validar los resultados producidos por esta técnica se evaluaron los niveles de turbidez en diferentes cursos de agua en las comunas de estudio. El número de muestras correspondió a 34 subcuencas (figura 5).

Los valores generales indican altos niveles de turbidez para Lanco y también en algunos puntos de Corral, sin embargo, la comuna de Pucón presenta niveles muy reducidos de turbidez. De acuerdo a los niveles de turbidez identificados por subcuenca y los valores mínimos entre-

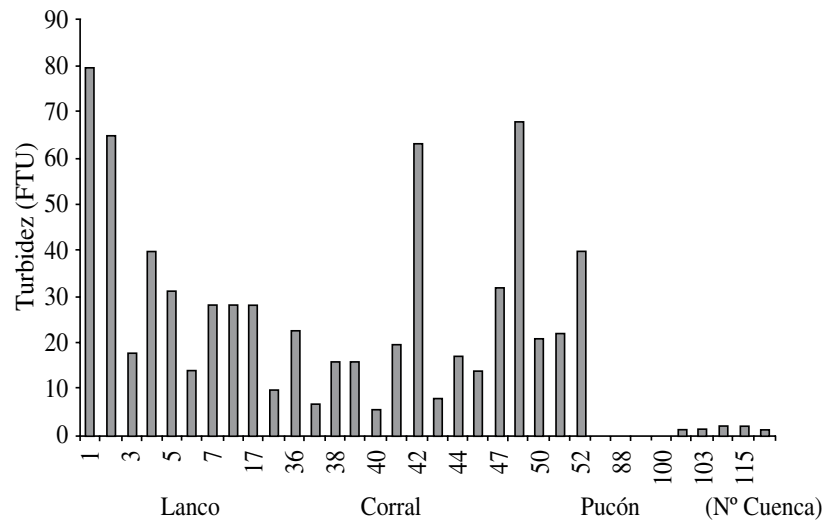

Figura 5. Niveles de turbidez medidos en subcuencas de las comunas de Lanco, Corral y Pucón.

Turbidity levels measured in subriver basins of Lanco, Corral and Pucón Counties.

gados para esas mismas áreas por el modelo, muestran un coeficiente de correlación (r) de 0,67 $(P<0,05)$.

\section{DISCUSIÓN Y CONCLUSIONES}

Los indicadores parciales entregan una aproximación para evaluar el riesgo de la degradación de los recursos generadores de agua de calidad. Su integración mediante la regla de combinación ayuda a tener una concepción agregada de este riesgo en un ámbito geográfico determi- 
nado, y relaciona la turbidez de los cursos de agua con la influencia determinada por el uso preferente del suelo (Keithley 2000), como es indicativo de las actividades agroganaderas en la comuna de Lanco.

Esta metodología posee la ventaja de integrar múltiples variables hidrológicas y demuestra flexibilidad para el desarrollo de modelos ambientales, en la cual la información subjetiva debe ser correlacionada tal como lo describen Reynolds et al. (1996) y Dai et al. (2004). Los resultados obtenidos a través de esta técnica son de relativa fácil aplicación y permiten integrar o modificar el conocimiento base ya establecido. Esto permite el ajuste en los resultados mediante la búsqueda de nueva evidencia o mediante el perfeccionamiento de ésta.

La técnica empleada facilita la identificación de áreas potenciales que pueden ser utilizadas para desarrollar estudios o prospecciones más profundas, constituyendo de esta manera una herramienta de interés para la planificación territorial. Bajo la hipótesis propuesta, se logra la identificación de áreas de provisión de agua de calidad, haciendo posible la discriminación (priorización) de aquellas zonas con mayores niveles de probabilidad, permitiendo además el manejo de la incertidumbre en la información (Reynolds et al. 1996, Ocampo-Duque et al. 2006). También posibilita estudiar y simular el efecto sobre la calidad del agua por cambios de uso de la tierra o construcción de infraestructura vial.

Si bien la técnica se aplicó al concepto agua de calidad mediante el indicador de turbidez, es extensible a otros servicios ambientales del territorio. El desafío de una gestión eficiente del territorio que comprenda un mejor manejo y toma de decisiones de los recursos naturales del país demandará cada vez más la adopción y desarrollo de políticas, estrategias e instrumentos económicos basados en las potencialidades y restricciones de cada unidad espacial, lo cual abre una posibilidad para la utilización de las técnicas y modelos propuestos en este trabajo.

Algunas de las debilidades de estas técnicas dicen relación con la subjetividad en el tratamiento de las variables utilizadas, tales como las desarrolladas mediante clasificación difusa. Debido a estas razones, se hace necesario un conocimiento acabado de las relaciones causa-efecto producido por las variables sobre el recurso hídrico con el propósito de mejorar las estimaciones del modelo.

A pesar de estos elementos, se destaca la aplicación de estas técnicas poco utilizadas en el país, que pueden llegar a constituir una herramienta útil en el manejo de recursos naturales, en particular para integración de múltiples variables en un contexto territorial y en la toma de decisiones en torno a procesos ecológicos complejos.

\section{AGRADECIMIENTOS}

Este trabajo fue elaborado sobre la base del proyecto sobre modelamiento de la oferta de bienes y servicios ambientales, proyecto financiado por la DID de la UACh, Código S-200435.

\section{REFERENCIAS}

Banzhaf S, J Boyd. 2004. Indexes and indicators of ecosystem services. Washington DC, USA. Resources for the Future. $30 \mathrm{p}$.

Barton D, D Faith, G Rush, JO Gjershaug, M Castro, M Vega, E Vega. 2003. Spatial prioritization of environmental service payments for biodiversity protection: Decision-making models for evaluating cost-effectiveness of conservation priorities using alternative biodiversity indicators. Report SNR 4746/2003. Norwegian Institute for Water Research. Consultado 05 dic. 2007. Disponible en http://www.inbio.ac.cr/ ecomapas/bioindicadores/paginas_es/Bioindicators.pdf

Beaudry P, B Floyd, B L'Hirondelle. 2003. Stream Crossing Quality Index (SCQI) Survey for 8 Sub-basins of Tree Farm License \#30 Prince George Forest District. Consultado 10 dic. 2007. Disponible en http://www.for.gov.bc.ca/hfd/ library/FIA/2003/FIA-03-04-0132a.pdf

Boyd J. 2004. What's nature worth? Using indicators to open the black box of ecological valuation. Washington DC, USA. Resources for the Future. 5 p.

Boyd J, L Wainger. 2003. Measuring Ecosystem Service Benefits: The use of landscape analysis to evaluate environmental trades and compensation. Discussion Paper 02-63. Washington DC, USA. Resources for the Future. 156 p.

CCAD-PNUD/GEF 2002. Guía metodológica de valoración económica de bienes, servicios e impactos ambientales. Un aporte para la gestión de ecosistemas y recursos. Consultado 15 nov. 2007. Disponible en http://xsei.centrogeo.org.mx/vedet/ biblioteca/val_eco_deg/VE_Valoraci\%F3n CBM.pdf.

CONAF (Corporación Nacional Forestal, CL), CONAMA (Comisión Nacional del Medio Ambiente, CL). 1999a. Catastro y evaluación de recursos vegetacionales nativos de Chile, Informe Nacional con Variables Ambientales. Proyecto CONAF-CONAMA-BIRF, Santiago, Chile. 88 p.

CONAF (Corporación Nacional Forestal, CL), CONAMA (Comisión Nacional del Medio Ambiente, CL). 1999b. Proyecto catastro y evaluación de los recursos vegetacionales nativos. Sistema y Base de Datos versión 2.0cli. Aplicación Arc View en CD.

Dai J, S Lorenzato, D Rocke. 2004. A knowledge-based model of watershed assessment for sediment. Environmental modeling and software 19 (2004): 423-433.

Daniels B, D McAvoy, M Kuhns, R Gropp. 2004. Managing forests for water quality: forest roads. Utah State University. Consultado 15 dic. 2007. Disponible en http://extension.usu. edu/files/ publications/factsheet/NR_FF_010.pdf

Durst J, J Ferguson. 2000. Buffer strip function and design. An annotated bibliography. Alaska Department of Fish and Game, Habitat and Restoration Division. Consultado 01 dic. 2007. Disponible en http://forestry.alaska.gov/ pdfs/1LitBufferDesign8-7-00.pdf

Elliot W, D Hall, S Graves. 1998. X-DRAIN and XDS: a simplified road erosion prediction method. Rocky Mountain Research Station, USDA Forest Service. Consultado 04 oct. 2007. Disponible en http://www.fs.fed.us/rm/pubs_other/ rmrs_1998_elliot_w001.pdf 
Gallo K, S Lanigan, P Eldred, S Gordon, Ch Moyer. 2005. Northwest Forest Plan-the first 10 years (1994-2003): preliminary assessment of the condition of watersheds. Gen. Tech. Rep. PNW-GTR-647. Portland, OR. U.S. Department of Agriculture, Forest Service, Pacific Northwest Research Station. 133 p.

Hilliard C, S Reedyk. 2000. Soil texture and water quality. Water quality matters. PFRA- Agriculture and Agri-Food Canada. Consultado 18 oct. 2007. Disponible en http://www.agr. gc.ca/pfra/water/facts/soiltexe.pdf

Keithley C, 2000. Evaluating stream and watershed conditions in Northern California. Sacramento, CA. California Department of Fish and Games. 17 p.

Lee P, C Smyth, S Boutin. 2004. Quantitative review of riparian buffer width guidelines from Canada and the United States. Journal of Environmental Management 70 (2004): 165-180.

Morales, C S. Parada. 2005. Pobreza, desertificación y degradación de los recursos naturales. Libros de la CEPAL N ${ }^{\circ} 87$. Santiago, Chile. CEPAL. 267 p.

NCWAP (North Coast Watershed Assessment Program, US). 2001. North Coast Watershed Assessment Program Methods Manual (draft). Sacramento, CA. NCWAP. 108 p.

Ocampo-Duque W, N Ferre-Huguet, J Domingo, M Schuhmacher. 2006. Assessing water quality in rivers with fuzzy inference systems: A case study. Environment International 32 (6): 733-742.

Reynolds K, M Saunders, M Foster, R Olson, Schmoldt, D Latham, B Miller, J Steffenson. 1996. A knowledge-based information management system for watershed analysis in the Pacific Northwest US. AI Applications 10: 9-22.

Saaty TL. 1977. A Scaling Method for Priorities in Hierarchical Structures. J. Math. Psychology 15: 234-281.

Schlatter J, V Gerding, J Adriazola 1995. Sistema de ordenamiento de la tierra. Herramienta para la planificación forestal aplicada a la X Región. Serie Técnica, Facultad de Ciencias Forestales. Valdivia, Chile. Universidad Austral de Chile. 93 p.

Schlatter J, V Gerding, J Adriazola. 1997. Sistema de ordenamiento de la tierra. Herramienta para la planificación forestal aplicada a la X Región. Serie Técnica, Facultad de Ciencias Forestales. Valdivia, Chile. Universidad Austral de Chile. 81 p.

Schmoldt DL, HM Rauscher. 1996. Building Knowledge-Based Systems for Natural Resource Management. New York, USA. Chapman and Hall. 408 p.

Sheridan G, HB So. 2001. Predicting the effect of slope gradient on soil erosion rates for steep landscapes. In Ascough JC and DC Flanagan eds. Soil erosion research for the 21st century, Proc. Int. Symp. (3-5 January 2001, Honolulu, HI, USA). St. Joseph. p. 451-454.

USDA Forest Service. 1999. Roads analysis: Informing decisions about managing the national forest transportation system. Misc. Rep. FS-643. Washington DC, USA. US Dept. of Agriculture Forest Service. 222 p.

Zadeh LA. 1965. Fuzzy Sets. Information and Control 8: 338-353. 